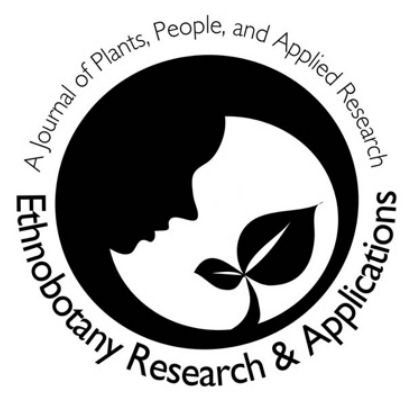

\title{
Phytodiversity and Ethnobotanical Features of Plants of Shahbaz Garhi Mardan, Khyber Pakhtunkhwa, Pakistan
}

\author{
Sadia Parveen, Lal Badshah, Asad Ullah, Syed Ghias Ali, \\ Syed Mukaram Shah, Usman Ali, Akhtar Zaman and Sara \\ Hamid Siddiqui
}

\author{
Correspondence \\ Sadia Parveen*, Lal Badshah, Asad Ullah, Syed Ghias Ali, Syed Mukaram Shah, Usman Ali, Akhtar Zaman \\ and Sara Hamid Siddiqui \\ Center of Plant Biodiversity, University of Peshawar, Khyber Pakhtunkhwa 25120, Pakistan, Peshawar Pakistan \\ ${ }^{*}$ Corresponding Author: sadia.bsbot12@gmail.com
}

Ethnobotany Research \& Applications 22:30 (2021)

\section{Databases and Inventories}

\begin{abstract}
Background. Plants provide food, clothing, shelter, medicines, fodder, fuel wood and ecosystem services. The floristic diversity of any area plays an important role in the sustainable livelihood and food security of the inhabitants of that area. The people of the research area Shahbaz Garhi, District Mardan are living in the far flung and backward area. They are dependent on plants and plants products for supporting their livelihood and other needs. They obtain food, fuel, timber, medicines and fodder from plants and also use plants for ornamental purposes. Since the area has a rich flora which was not previously documented. Similarly, the inhabitants use these plants for many purposes. Therefore, the present study was planned to document the flora and its local uses and to know how the local people use these plant natural resources to support their livelihood.
\end{abstract}

Methods. Thorough collection of plants was made from the research area during 2019-2020. Plants were collected and the related data was documented on the spot in the field. Identification was carried out with the help of authentic literature. The ethnobotanical data was collected through questionnaires and 50 informants were interviewed, whose age was ranging from 20-60 years. Standard procedures were adopted for ethnobotanical information collection which includes (Stijfhoorn 1996-1997) and (Martin 2004) procedures and further data authentication was made by adopting artifact (ex-situ) and inventory (in-situ) methods.

Results. A total of 85 species belonging to 42 families were recorded. Out of 42 families, 35 families were dicots, 5 were monocots and 2 families were gymnosperms. The dominant family was Asteraceae comprised of 7 genera (9.09 \%) and 7 species (8.23 \%) followed by Poaceae, Fabaceae and Amaranthaceae with 5 species each, while Cupressaceae, Myrtaceae, Oleaceae, Rhamnaceae, Salicaceae, Solanaceae and Verbenaceae with 3 species each and the remaining families were represented by 2 or less species. The life form class was dominated by Therophytes comprised of 31 spp. (36.4\%) followed by Nanophanerophytes 24 spp. (28.2 \%) and Chamaephytes 11 spp. (12.9 \%). The leaf size spectra showed that Microphylls was dominant with 25 spp. (29.4\%), followed by Mesophylls with 24 spp. (28.2\%), while the Nanophylls with 12 spp. (14.1\%) and Leptophylls with 10 spp. (11.7 \%). The habit of plants showed that 43 species (50.5\%) were herbs, 28 species (32.5\%) were trees, and 14 spp. (16.4 \%) were shrubs. 
The ethnobotanical analysis of 58 spp. showed that medicinal plants were dominant with 44 spp. (75.8\%) followed by fodder 27 spp. (46.5 \%), fuel wood 21 spp. (36.2 \%), ornamental 15 spp. (27.5\%), food 13 spp. (22.4 \%) and timber 11 spp. (18.9 \%). All the 85 species were evaluated for conservation status. During the conservation status studies it was found that 1 species (1.17 \%) i.e., Ailanthus altissima was endangered, 12 species (14.1\%) were least concern, and $72(84.7 \%)$ species were not Eealuated. The indigenous species were $34(40 \%)$ and introduced were $51(60 \%)$. Among the total species impacts of 51 introduced species were studied, in which most species were noted for interfering local flora $28(54.90 \%), 9(17.64 \%)$ for replacing local flora, $6(11.76 \%)$ as weeds /interfering crops, 2 (3.92\%) were allelopathic and 6 (11.76\%) were without any impacts.

Conclusion. It was revealed during the present study that the area has a rich diversity of plants and the people of the area use these plants for medicinal, fodder, fuel wood, ornamental, food, and timber purposes. The people of the area use these plants and their products to support their livelihood and other needs.

Key words. Phytodiversity, ethnobotanical features, Shahbaz Garhi, Mardan, Pakistan.

\section{Background}

Pakistan is a developing country, and the people are dependents on plants for fulfilling their daily needs (Ahmad 2007, Shinwari \& Gillani 2003). According to (Ali 2008) Flora is sum total of species in a specific area, which are peculiarity of an ecological period, while ethnobotany is defined as "the study of utilitarian relationship between human being and vegetation in their environment" (Harshberger 1896).Vegetation is the qualitative expression of plants of an area In spite of great importance, out of approximately 30 million living species only 1.75 million living species of the world have been described so for (Ahmad et al. 2019). Usually, floristic listing helps in identification and nomenclature of species (llyas et al. 2013). Since no such previous studies are carried out on the floristic and ethnobotanical uses of the plants of the research area. Therefore, the present study is aimed to document the floristic composition and ethnobotanical uses of plants growing in the area. The total area is 162085 hectares, in which 112790 hectares is cultivated and 49295 hectares is uncultivated including forest (7938 hectares), while the rest is not suitable for cultivation. Research was conducted on the ethnomedicinal uses of plants of Gujjar and Bakerwal community in Gulmarg Mountainous Region of Kashmir Himalaya. Total 60 plant species were collected belonging to 56 genera and 35 families in which Asteraceae family was found dominant. Taraxicum officinale, Achilla millfelium, Chenopodium album, Phytollaca acinosa, Ajuga bracteosa and Cannabis sativa were found most important (Jan et al. 2021). Following the same work, the ethnobotanical survey was conducted about the uses of plants at Kumrat valley, District Upper Dir Pakistan and it was found that the local communities, of Upper Dir depends upon plants for nutrition, medicine, and monetary benefits. Total 50 plant species were reported belonging to 30 families and their organic products were identified to show its market value and other uses (Ahmad et al. 2021). The traditional ethnobotanical uses of wild plants Dawarian and Ratti Gali areas of District Neelum, Azad Jammu and Kashmir Pakistan were studied which also resembles our work. It was found that these plants were used for different purposes i.e., as food, vegetables, fodder, fuel, shelter, timber, and herbal medicines. These plants were also used for treatment of different diseases such as joint pain, cancer, cardiovascular disorders, and skin diseases. It was also found that Berberis lycium, Ajuga bracteosa, Saussurea lapa and Jurinea dolomiaea are severely threatened (Ajaib et al. 2021). Also, another study was conducted on the traditional uses of plants by indigenous communities for veterinary practices in Kurram District, Pakistan. Total 81 plants were reported belonging to 43 families in which Asteraceae and Lamiaceae were dominant. It was found that these plants were used for digestive diseases, skin problems, as anthelmintic and to treat diarrhea. It is important to mention that such studies can be helpful in identification of active ingredients (Ali et al. 2019). It was noted during the present research that the land of the current research area is mostly used for agricultural purposes, known as best agricultural area in the country. It is mostly suitable for cultivation of sugarcane and tobacco, also called land of sugarcane and tobacco. The major crops grown in the area wheat, sugar cane, tobacco, maize, rice, rape seed and mustard. Fruits and vegetables are also grown. The soil ranged from sandy loam to clay and is suitable for growing various agricultural crops and various tree species. The geology of the District shows that the area can be divided into two parts i.e., South Western Plain area and North Eastern hilly part. The plain area consists of alluvial soil covering 100 of meters thickness and the mountains are part of rocks of Shewa Shahbaz Garhi. The Shahbaz Garhi complex is divided into acidic and basic rock types. Acidic rocks are microporphyry, porphyritic granite, aegirine riececkite porphyry, riececkite gneiss. The acidic rocks are intruded by basic rocks such as metagabbro, metadolorite and local quartz monozonite. Minerals in the basic rocks include hastingsite, clinopyroxene, orthopyroxene, biotite and epidote while the acidic rocks have orthoclase, plagioclase and ferromagnesian minerals. 


\section{Hypothesis}

The main aim of this study was to find out that being far flung area of the country, the local inhabitants are depending upon directly or indirectly on the plant natural resources growing in the research area.

\section{Materials and Methods}

\section{Study Area}

The name Mardan means the "Land of Brave Men". Historically the name was from old Sanskrit "Amar Daan". Mardan was a city in the Mardan District of Khyber Pakhtunkhwa, located in the Valley of Peshawar and second largest city of Khyber Pakhtunkhwa after Peshawar city. Mardan covering an area of $1632 \mathrm{~km}^{2}$ and with a population of 2,373,061. District Mardan lies from $34^{\circ} 05^{\prime}$ to $34^{\circ} 32^{\prime} \mathrm{N}$ and $71^{\circ} 48^{\prime}$ to $72^{\circ} 25^{\prime} \mathrm{E}$, bounded on the north by Buner District, on the east by Swabi, on the south by Nowshera District and on the west by Charsadda District.
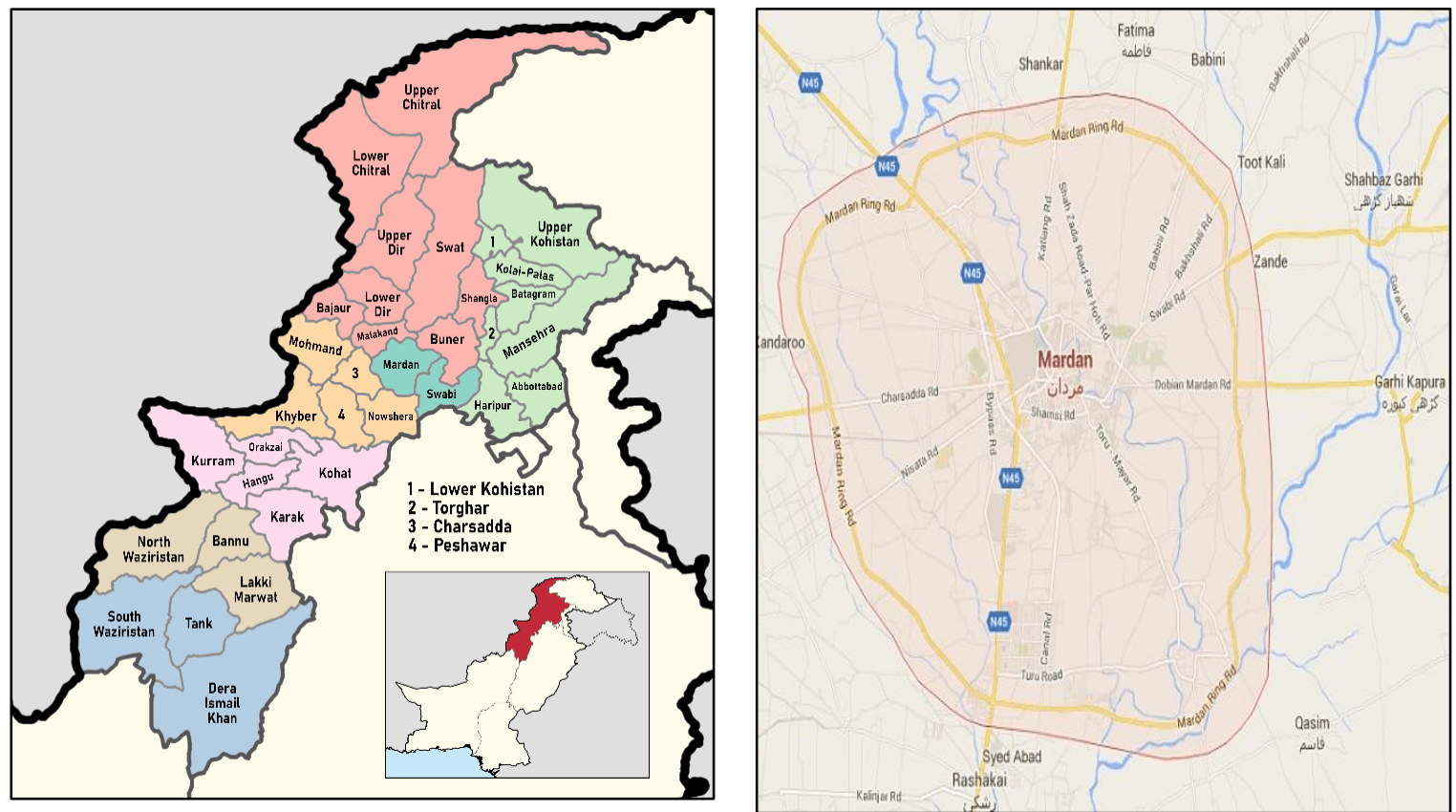

Figure 1. Map showing the study area Shahbaz Garhi, District Mardan, Khyber Pakhtunkhwa, Pakistan

Shahbaz Garhi is a village and historic site located in Mardan District in Khyber Pakhtunkhwa Province of Pakistan. Shahbaz Garhi is located in the southwest of the district Mardan at 34² $12^{\prime} \mathrm{N}, 7^{\circ} 16^{\prime} \mathrm{E}$ and an altitude of 293 masl. It is about $12 \mathrm{~km}$ from Mardan city. It has mountains, green and open fields and a small river flowing in the center of village.

In old times all these facilities made it attractive for the army and travelers to dig in their tents here, stay for few days and organize their further strategy. The historic stones of Ashoka, and other sites like Mekha Sanda are worth visiting. The common trees are Prosopis glandulosa, Ziziphus mauritiana, Vachellia nilotica. Cupressus sempervirens, Thuja orientalis, Pinus roxburghii, Phoenix dactylifera. The most common shrubs were Juniperus communis, Agave americana, Nerium oleander, Opuntia littoralis and Hibiscus rosa-sinensis, Dodonaea viscosa. Lantana camara while common Herbs were Mirabilis jalapa, Oxalis corniculata, Papaver rhoeas, Papaver somniferum and Datura stramonium.

\section{Climate}

The summer season is extremely hot with steep rise of temperature from May, June, July, August and up to September. The temperature reaches to its maximum in the month of June i.e., $43.5^{\circ} \mathrm{C}\left(110.3{ }^{\circ} \mathrm{F}\right)$. Due to intensive cultivation and artificial irrigation the tract is humid, and heat is oppressive. The coldest months are December and January. The mean minimum temperature recorded for month of January is $0.5^{\circ} \mathrm{C}\left(32.9{ }^{0} \mathrm{~F}\right)$. Maximum rainfall recorded for the month of August is $12.8 \mathrm{~mm}$. The maximum humidity has been recorded in December i.e., $73.33 \%$. The plant assemblage of a region is a function not only of time but also of altitude, slope, latitude, aspect, rainfall, and humidity which play a role in its formation and composition (Kharkwal et al. 2005). 


\section{Irrigation system}

Many exotic species are introduced in the irrigated areas. Generally, streams flow from north to the south and most of the streams drain into Kabul River. Kalpani, an important stream of the district rises in the Baizai and flowing southwards join Kabul River. Other important streams are Baghiari Khawar on the west and Muqam Khawar, coming from Sudham valley and Naranji Khawar from the Narangi on the left. Mardan district was broadly divided into two parts, northeastern hilly area and southwestern plain. In the district, the highest points in these hills are Garo or Pato (1816 masl) and Pajja or Sakra (2056 masl). The southwestern half of the district is mostly composed of fertile plain with low hills strewn across.

\section{Collection, processing, and identification}

To collect plants and to prepare a complete floristic list of plants, frequent visits were made to different localities in the research area Shahbaz Garhi, District Mardan, Khyber Pakhtunkhwa during 2019-20. Plants were collected during the months of June and August. The collected plants were pressed in the newspapers to make them moisture free and were dried. Plants were then identified by the flora of Pakistan (Nasir and Ali 1970-1989, Ali and Nasir 1989-1991, Stewart 1972, Ali and Qaiser 1993-2021).

Plants were further identified and confirmed at Herbarium Department of Botany, University of Peshawar, Pakistan. After that the plants were mounted on Herbarium sheets. Voucher specimens were submitted to the Herbarium, Department of Botany (PUP). Biological spectra including life form and leaf size classes were classified after (Raunkiar 1934, Hussain 1989).

\section{Phytodiversity and check listing}

The plants were grouped into divisions, families and a floristic list was prepared in chronological order including botanical name, local names, life form, leaf size and habit.

\section{Ethnobotanical data retrieval}

Ethnobotanical information werewas collected by field visits to eight different localities and villages of Shahbaz Garhi including Khairabad, Hosai, Bikhan, Gulyara, Mohib Banda, Bala Garhi, Kamal Khan Garden and Tourist shed at Asoka Remains Shahbaz Garhi. Data regarding ethnobotanical uses of species was collected through questionnaire followed (Ali and Qaiser 2009, Ullah 2014). A total of 50 respondents were randomly interviewed through questionnaire. The age of the respondents was ranging from 20-60 years. The (Stijfhoorn 1996-1997, Martin 2004) procedures were adopted for the collection of information of plant usage i.e., Artifact (ex-situ) and inventory (in-situ) methods were adopted for authentication of data.

\section{Conservation, indigenous and introduced status and impacts studies}

For determination of Conservation status IUCN (2019) Red List categories version 14 were followed (extinct, extinct in the wild, critically endangered (CR), endangered (EN, Vulnerable (VU), near threatened (NT), least concern (LC), data deficient (DD) and not evaluated (NE). Indigenous and introduced status was also determined by consulting Flora of Pakistan and other related literature. The impacts of the collected species were recorded through personal observation and interviewing the local people.

\section{Results and Discussion}

\section{Phytodiversity studies}

During the present study 85 plant species of 42 families were documented. 2 families were gymnosperms (4 spp.) and 40 families were angiosperms. 5 families were monocots (10 spp.) and 35 families were dicots (71 spp.)

Dominant gymnosperms family was Cupressaceae (2 spp.) followed by Pinaceae (1 sp.). Among the 5 families of monocots, Poaceae was the dominant family with $5 \mathrm{spp}$. followed by Amaryllidaceae (2 spp.). Asteraceae was the dominant family among dicots with $7 \mathrm{spp}$. followed by Amaranthaceae and Fabaceae with 5 spp. each (Table 1). Some other workers also conducted similar studies including (Sher et al. 2011) from different regions of Khyber Pakhtunkhwa, Pakistan. Seven (7) species of Asteraceae and 6 of Poaceae as dominant families were reported from district Toba Tek Singh, Pakistan (Qureshi et al. 2009). 
Table 1. Percent distribution of plant families in the research area

\begin{tabular}{|c|c|c|}
\hline Families & No of species & Percentage \\
\hline Asparagaceae & 1 & 1.18 \\
\hline Arecaceae & 1 & 1.18 \\
\hline Amaryllidaceae & 2 & 2.35 \\
\hline Apocynaceae & 1 & 1.18 \\
\hline Araliaceae & 1 & 1.18 \\
\hline Amaranthaceae & 5 & 5.9 \\
\hline Asteraceae & 7 & 8.24 \\
\hline Adiantaceae & 1 & 1.18 \\
\hline Apiaceae & 2 & 2.35 \\
\hline Asclepiadaceae & 2 & 2.35 \\
\hline Bignoniaceae & 1 & 1.18 \\
\hline Bombaceae & 1 & 1.18 \\
\hline Boraginaceae & 1 & 1.18 \\
\hline Brassicaceae & 1 & 1.18 \\
\hline Cuppressaceae & 3 & 3.53 \\
\hline Commelinaceae & 1 & 1.18 \\
\hline Cannaceae & 1 & 1.18 \\
\hline Cactaceae & 1 & 1.18 \\
\hline Cucurbitaceae & 2 & 2.35 \\
\hline Euphorbiaceae & 1 & 1.18 \\
\hline Fabaceae & 5 & 5.88 \\
\hline Liminaceae & 2 & 2.35 \\
\hline Moraceae & 4 & 4.70 \\
\hline Myrtaceae & 3 & 3.52 \\
\hline Meliaceae & 1 & 1.18 \\
\hline Malvaceae & 1 & 1.18 \\
\hline Nyctiginaceae & 2 & 2.35 \\
\hline Oleaceae & 3 & 3.52 \\
\hline Oxalidaceae & 1 & 1.18 \\
\hline Papaveraceae & 2 & 2.35 \\
\hline Pinaceae & 1 & 1.18 \\
\hline Poaceae & 5 & 5.88 \\
\hline Rosaceae & 2 & 2.35 \\
\hline Rhamnaceae & 3 & 3.52 \\
\hline Rutaceae & 1 & 1.18 \\
\hline Silacaceae & 3 & 3.52 \\
\hline Solanaceae & 3 & 3.52 \\
\hline Sapindaceae & 1 & 1.18 \\
\hline Simaroubaceae & 1 & 1.18 \\
\hline Tamaricaceae & 1 & 1.18 \\
\hline Verbenaceae & 3 & 3.52 \\
\hline Zygophyllaceae & 1 & 1.18 \\
\hline Total & 85 & 100 \\
\hline
\end{tabular}

Raunkiaerian classification showed that Therophytes (31 spp., $36.4 \%$ ) was the dominant life form followed by Nanophanerophytes (24 spp., 28.2\%), Chamaephytes (11 spp., 12.9\%), Mesophanerophytes (11 spp., 12.9\%), Hemicryptophytes (2 spp., $2.3 \%$ ), Geophytes 5 spp., $5.8 \%$ ) and Megaphanerophytes (1 sp., $1.1 \%$ ). Leaf size spectra showed that Microphylls with (25 spp., 29.4\%) and Mesophylls with (24 spp., $28.2 \%$ ) were the dominant groups. They were followed by Nanophylls with (12 spp., $14.1 \%$ ), Leptophylls (10 spp., 11.7\%), Megaphylls (8 spp., $9.4 \%$ ), Macrophylls (4 spp., $4.7 \%$ ) and Aphyllous (2 spp., $2.3 \%)$. The flora the research area was dominated by herbs with 43 spp. (50.5\%) followed by trees with 28 spp. (32.5 $\%)$ and shrubs with 14 species (16.4\%) (Table 2, Figs. 2 and 3).

\section{Ethnobotanical studies}

According to previous studies about $80 \%$ of the population of the developing countries relies on herbal medicines as a primary health care need (Mukerjee and Wahil 2006). In current research work 58 plants were recorded from study areas which were ethnobotanically used by local inhabitants in different ways. Among them, 44 were medicinal (75.8\%), fodder were 27 spp. (46.5 \%), fuel wood were 21 spp. (36.2\%), ornamental were 15 spp. (27.5\%), food were 13 spp. (22.4\%) and timber were 11 spp. (18.9\%) (Table 2, Fig. 4).

\section{Conservation, indigenous and introduced status and impacts studies}

All the 85 species were evaluated by using IUCN (2019) red list categories for determination of conservation status. It was found that majority of species i.e., $72 \mathrm{spp}$. (84.7\%) were not evaluated while $12 \mathrm{spp}$. (14.1\%) were least concern. One species (1.17 \%) was endangered i.e., Ailanthus altissima. This evaluation showed that there is no major conservation issue in the area because the area is limited, and majority of the species are not evaluated or are species of least concern. (Table 3).

The species were also evaluated for its indigenous (Ind.) and exotic (Int.) status by consulting Flora of Pakistan and other Florae of the world. It was concluded that $34 \mathrm{spp}$. (40\%) were indigenous, and $51 \mathrm{spp}$. (60\%) were introduced. This showed that the numbers of introduced species are more. These species are introduced for ornamental purposes, or these may be arrived as weeds in seeds of various crops including wheat and maize (Table 3).

The introduced species always have some impacts on the local flora, crops and ecosystem. Among the total 51 introduced plants it was found that majority of the species 28 (54.90\%) are interfering the local flora and thus increasing the chances of replacement of the local flora, similarly, 9 species (17.64\%) were involved directly in replacing local flora. Some species are known as weeds and they compete in many ways with the local crops, in the present study 6 (11.76\%) species were found as weeds. Some plants release chemical compounds which are menacing to other species, and they damage other plants by releasing chemical compounds. In this research study $2(3.92 \%)$ species were found allelopathic. In this study 6 (11.76\%) species were also found with no significance adverse impacts (Table 3 ). 
Table 2. Ethnobotanical profile of plants of Shahbaz Garhi

\begin{tabular}{|c|c|c|c|c|c|c|}
\hline Botanical name & $\mathrm{Fd}$ & $\mathrm{Tm}$ & $\mathrm{Fu}$ & Md & Fod & Orn \\
\hline Agave americana $\mathrm{L}$. & - & - & - & - & - & + \\
\hline Allium sativum $\mathrm{L}$. & + & - & - & + & + & - \\
\hline Achyranthes aspera L. & - & - & - & + & - & + \\
\hline Aerva javanica (Burmf.) Schult & + & - & - & - & - & - \\
\hline Adiantum capillus-veneris L. & + & - & - & + & - & - \\
\hline Ailanthus altissima (Mill.) Swingle & + & + & + & + & - & - \\
\hline Bombax ceiba L. & - & - & + & - & + & - \\
\hline Bauhinia variegata (L.) Benth & + & - & + & + & - & - \\
\hline Broussonetia papyrifera (L.) Vent & - & - & + & + & - & - \\
\hline Bougamvillea glabra Choisy & - & - & - & - & - & + \\
\hline Cupressus sempervirens L. & - & + & - & + & - & + \\
\hline Cenchrus cilliaris L. & + & - & - & - & - & - \\
\hline Conyza canadensis (L.) Cronqui & + & - & - & + & - & - \\
\hline Coriandrum sativum $\mathrm{L}$. & + & - & - & + & + & - \\
\hline Calotropis procera (Wild) R. Br. & - & - & - & + & - & - \\
\hline Canna indica $\mathrm{L}$. & - & - & - & + & - & + \\
\hline Cassia fistula L. & + & + & + & + & - & + \\
\hline Cucurbita pepo L. & - & - & - & - & + & - \\
\hline Citrus limon (L.) Osbeck & - & - & + & + & + & + \\
\hline Digera muricata (L.) & + & - & - & + & - & - \\
\hline Dodonaea viscosa (L.) Jacq. & - & - & + & + & - & - \\
\hline Datura metel L. & - & - & - & + & - & - \\
\hline Eruca sativa Mill. & + & - & - & + & + & - \\
\hline Euphorbia prostrata L. & - & - & - & + & - & - \\
\hline Eriobotrya japonica (Thunb.) & - & - & + & + & - & - \\
\hline Ficus carica Hausskn. ex. Boiss & + & - & + & + & + & - \\
\hline Ficus religiosa L. & - & - & + & + & - & - \\
\hline Hordeum murunum L. & + & - & - & - & - & - \\
\hline Helianthus annuus L. & + & - & - & + & + & + \\
\hline Heliotropium europaeum L. & + & - & - & + & - & - \\
\hline Hibiscus rosa-sinensis L. & - & - & - & - & - & + \\
\hline Imperata cylindrica (L.) P. Beauv & + & - & - & - & - & - \\
\hline Jasminum sambac Aito. & - & - & - & + & - & + \\
\hline Jasminum humile $\mathrm{L}$. & - & - & - & + & - & + \\
\hline Lactuca sativa L. & + & - & - & + & + & + \\
\hline Lantana camara L. & - & - & - & + & - & + \\
\hline Momordica charantia L. & - & - & - & + & - & - \\
\hline Mentha arvensis L. & - & - & + & + & - & - \\
\hline Morus alba L. & - & + & + & + & + & - \\
\hline Melia azedarach L. & + & + & + & + & - & - \\
\hline Nerium oleander $\mathrm{L}$. & - & - & - & + & - & + \\
\hline Ocimum basilicum L. & + & - & - & + & - & - \\
\hline Oxalis corniculata L. & + & - & - & + & + & - \\
\hline Pinus roxburghii Sarg. & - & + & + & + & - & - \\
\hline Phoenix dactylifera L. & - & + & + & + & + & - \\
\hline Phalaris minor $\mathrm{L}$. & + & - & - & - & - & - \\
\hline Parthenium hysterophorus L. & - & - & - & - & - & - \\
\hline Peganum harmata L. & - & - & - & + & - & - \\
\hline Psidium guajava $\mathrm{L}$. & + & - & + & - & - & - \\
\hline Papaver somniferum $\mathrm{L}$. & - & - & - & + & - & - \\
\hline Populus nigra L. & - & + & + & + & - & - \\
\hline Rosa indica Lindl & - & - & - & + & - & + \\
\hline Salix acmophylla Boiss & + & - & + & - & - & + \\
\hline
\end{tabular}




\begin{tabular}{|l|c|c|c|c|c|c|}
\hline Thuja orientalis L. & - & + & - & + & - & + \\
\hline Vicia sativa L. & + & - & - & - & - & - \\
\hline Ziziphus mauritiana Lam. & + & + & + & + & - & - \\
\hline Ziziphus nummalaria (Burm. F.) Weight & + & - & + & + & + & - \\
\hline Ziziphus jujuba Mill. & + & + & + & + & - & - \\
\hline Total & $\mathbf{2 7}$ & $\mathbf{1 1}$ & $\mathbf{2 1}$ & $\mathbf{4 4}$ & $\mathbf{1 3}$ & $\mathbf{1 6}$ \\
\hline Percentage & $\mathbf{4 6 . 5 \%}$ & $\mathbf{1 8 . 9 \%}$ & $\mathbf{3 6 . 2 \%}$ & $\mathbf{7 5 . 8 \%}$ & $\mathbf{2 2 . 4 \%}$ & $\mathbf{2 7 . 5 \%}$ \\
\hline
\end{tabular}

Key: Fd= Fodder, Md= Medicinal, Fu= Fuel, Tm= Timber, Fod= Food, Orn.= Ornamental; +: Yes, -: No

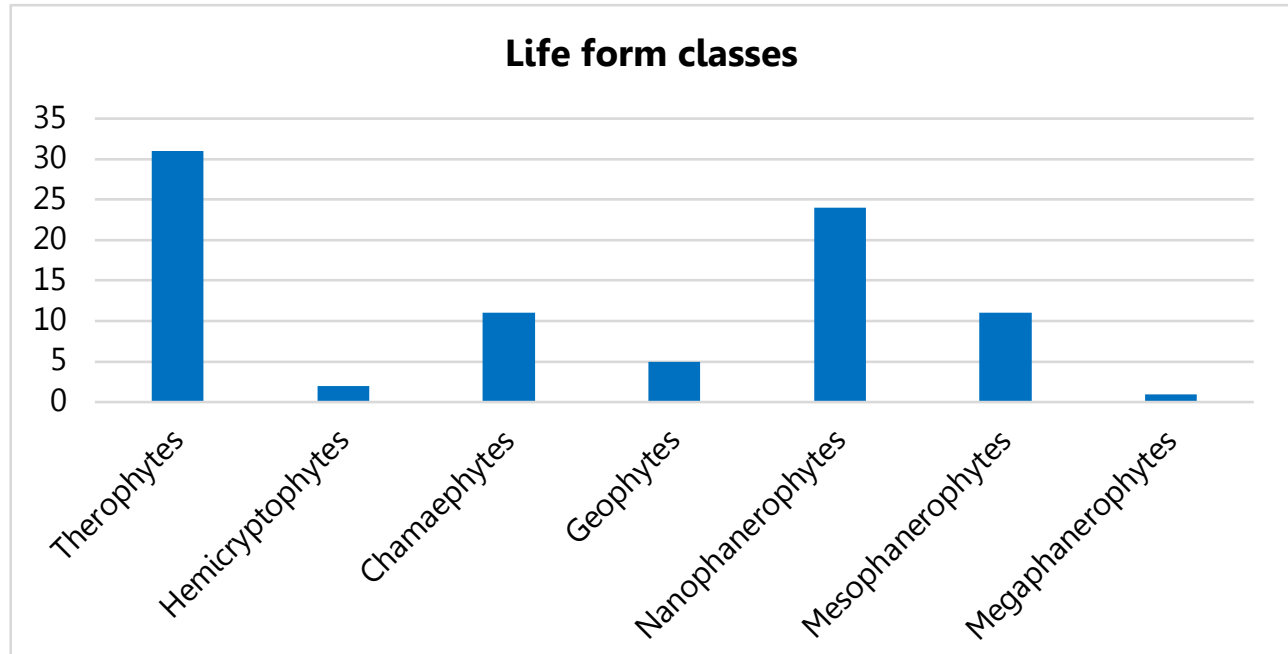

Figure 2. Life form classes of the plants growing in the area.

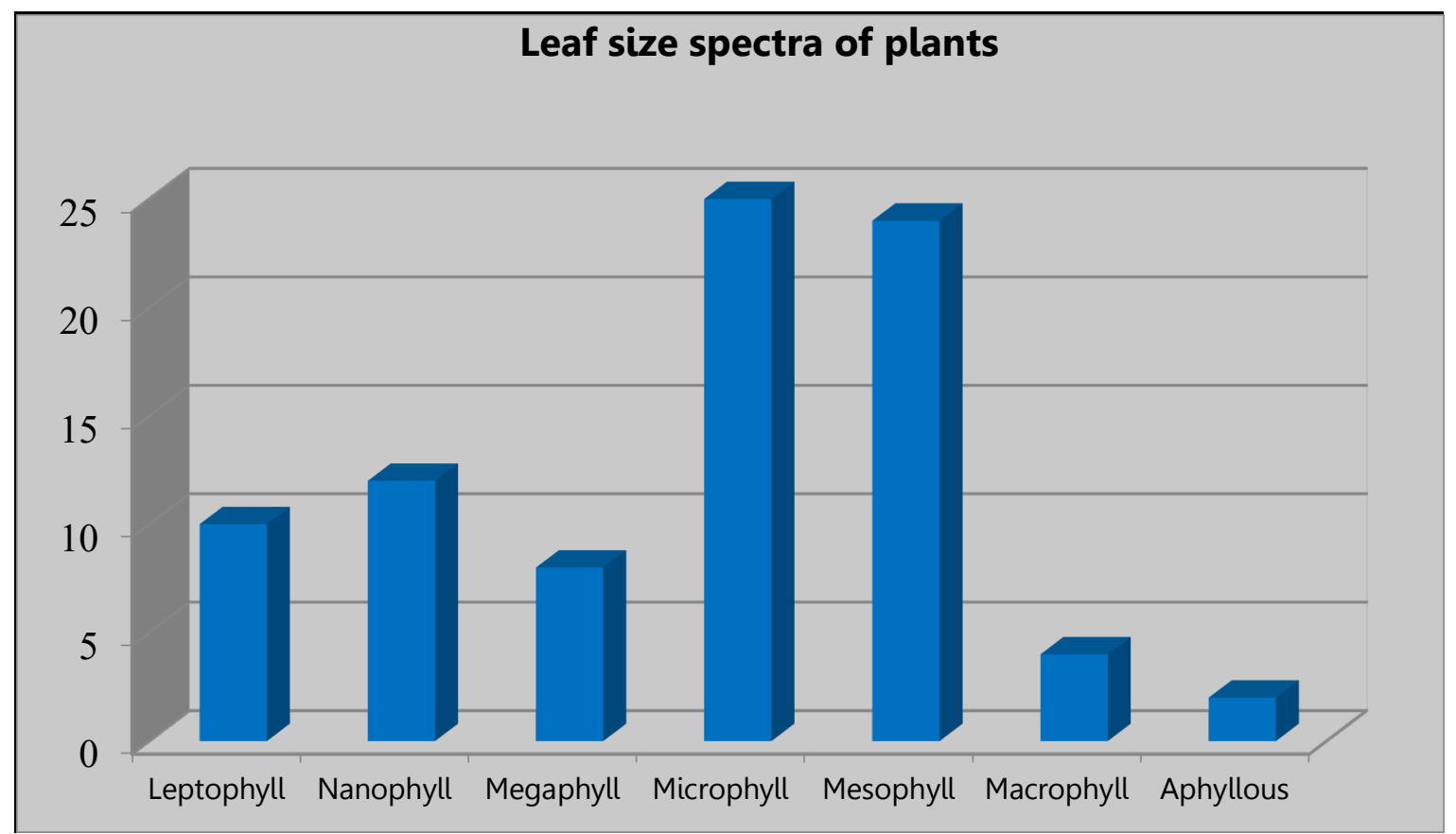

Figure 3. Leaf size classes of plants growing in the area. 
Table 3. Phytodiversity, biological spectrum, habit, conservation Ind./Int. status and impacts of the flora of Shahbaz Garhi

\begin{tabular}{|c|c|c|c|c|c|c|c|}
\hline Division\Family\Species & $\begin{array}{c}\text { Common } \\
\text { Name }\end{array}$ & Life form & Leaf Size & Habit & $\begin{array}{c}\begin{array}{c}\text { Conservation } \\
\text { status }\end{array} \\
\end{array}$ & $\begin{array}{c}\text { Status } \\
\text { Ind./Int. } \\
\end{array}$ & Impacts \\
\hline \multicolumn{8}{|l|}{ Gymnosperms } \\
\hline \multicolumn{8}{|l|}{ Cupressaceae } \\
\hline Juniperus communis L. & Gojar & $\mathrm{Np}$ & $\mathrm{L}$ & $S$ & $\mathrm{LC}$ & Ind. & - \\
\hline Cupressus sempervirens L. & Sarwa & Mesp & $\mathrm{L}$ & $\mathrm{T}$ & $\mathrm{NE}$ & Ind. & - \\
\hline Thuja orientalis L. & Maju & $\mathrm{Np}$ & $\mathrm{L}$ & $\mathrm{T}$ & $\mathrm{LC}$ & Int. & Replacement of local flora \\
\hline \multicolumn{8}{|l|}{ Pinaceae } \\
\hline Pinus roxburghii Sarg. & Nakhtar & $\mathrm{Np}$ & $\mathrm{N}$ & $\mathrm{T}$ & $\mathrm{LC}$ & Ind. & - \\
\hline \multicolumn{8}{|l|}{ Monocotyledons } \\
\hline \multicolumn{8}{|l|}{ Asparagaceae } \\
\hline Agave americana $\mathrm{L}$. & Zargira & $\mathrm{Ch}$ & Mes & $\mathrm{S}$ & $\mathrm{NE}$ & Int. & Replacement of local flora \\
\hline \multicolumn{8}{|l|}{ Arecaceae } \\
\hline Phoenix dactylifera L. & Kajoor & $\mathrm{Np}$ & Mic & $\mathrm{T}$ & $\mathrm{NE}$ & Ind. & - \\
\hline \multicolumn{8}{|l|}{ Amaryllidaceae } \\
\hline Allium sativum $\mathrm{L}$. & Ooga & $\mathrm{G}$ & Ap & $\mathrm{H}$ & $\mathrm{NE}$ & Int. & Allelopathic \\
\hline Allium siculum $\mathrm{L}$. & Oogakay & $\mathrm{G}$ & Ap & $\mathrm{H}$ & $\mathrm{NE}$ & Int. & Allelopathic \\
\hline \multicolumn{8}{|l|}{ Commelinaceae } \\
\hline Tradescantia pallida (Rose) D. R. Hunt & - & Th & $\mathrm{L}$ & $\mathrm{H}$ & $\mathrm{NE}$ & Int. & Replacement of local flora \\
\hline \multicolumn{8}{|l|}{ Poaceae } \\
\hline Bambusa vulgaris Schrad.ex J.C. Wendl & Bans & $\mathrm{Np}$ & Mes & $\mathrm{H}$ & $\mathrm{NE}$ & Int. & Interfering local flora \\
\hline Cenchrus ciliaris L. Mant. & Dandol & Th & $\mathrm{N}$ & G & $\mathrm{NE}$ & Ind. & - \\
\hline Imperata cylindrica (L.) P. Beauv & Drab & Hem & Mic & $\mathrm{H}$ & $\mathrm{NE}$ & Ind. & - \\
\hline Hordeum murinum $\mathrm{L}$. & Varbashaki & Th & $\mathrm{N}$ & $\mathrm{H}$ & $\mathrm{NE}$ & Int. & Invasive weed/Interfering crops \\
\hline Phalaris minor Retz. & Wakha & Th & Mic & $\mathrm{H}$ & $\mathrm{NE}$ & Ind. & - \\
\hline \multicolumn{8}{|l|}{ Dicotyledons } \\
\hline \multicolumn{8}{|l|}{ Apocynaceae } \\
\hline Nerium oleander L. & Gand derai & $\mathrm{Np}$ & Mic & $\mathrm{S}$ & $\mathrm{LC}$ & Int. & Interfering local flora \\
\hline \multicolumn{8}{|l|}{ Araliaceae } \\
\hline Hedera helix $\mathrm{L}$. & Prewatai & $\mathrm{Np}$ & Mes & C & $\mathrm{NE}$ & Int. & Interfering local flora \\
\hline
\end{tabular}




\begin{tabular}{|c|c|c|c|c|c|c|c|}
\hline Division\Family\Species & $\begin{array}{c}\text { Common } \\
\text { Name }\end{array}$ & Life form & Leaf Size & Habit & $\begin{array}{c}\text { Conservation } \\
\text { status }\end{array}$ & $\begin{array}{c}\text { Status } \\
\text { Ind./Int. }\end{array}$ & Impacts \\
\hline \multicolumn{8}{|l|}{ Amaranthaceae } \\
\hline Achyranthes aspera $\mathrm{L}$. & Puthkanda & Th & $\mathrm{N}$ & $\mathrm{H}$ & $\mathrm{NE}$ & Int. & Interfering local flora \\
\hline Aerva javanica (Burm. f.) Juss. & Sparai & $\mathrm{Ch}$ & Mic & $\mathrm{H}$ & $\mathrm{NE}$ & Int. & Interfering local flora \\
\hline Alternanthera sessilis L. & Azghakay & Th & Mic & $\mathrm{H}$ & $\mathrm{LC}$ & Int. & Interfering local flora \\
\hline Celosia cristata L. & Qarqara & Th & Mac & $\mathrm{H}$ & $\mathrm{NE}$ & Int. & Interfering local flora \\
\hline Digera muricata (L.) Mart. & Soor gulai & Th & Mic & $\mathrm{H}$ & $\mathrm{NE}$ & Ind. & - \\
\hline \multicolumn{8}{|l|}{ Asteraceae } \\
\hline Conyza canadensis (L.) Cronqui & $\begin{array}{l}\text { Naraiboty/ } \\
\text { Malooch }\end{array}$ & Th & $\mathrm{Na}$ & $\mathrm{H}$ & NE & Int. & Interfering local flora \\
\hline Echinops carnigerus DC. & Orejakai & $\mathrm{Ch}$ & Mes & $\mathrm{H}$ & $\mathrm{NE}$ & Ind. & - \\
\hline Helianthus annuus L. & Nawar paras & Th & Mes & $\mathrm{H}$ & $\mathrm{NE}$ & Int. & Replacing local crops \\
\hline Lactuca sativa $\mathrm{L}$. & Sallad pani & Th & Mic & $\mathrm{H}$ & $\mathrm{NE}$ & Int. & Interfering local flora \\
\hline Onopordum acanthium $\mathrm{L}$. & Wrijakai & $\mathrm{Ch}$ & $\mathrm{Na}$ & $\mathrm{H}$ & $\mathrm{NE}$ & Int. & Interfering crops \\
\hline Parthenium hysterophorus L. & Harzakay & Th & Ms & $\mathrm{H}$ & $\mathrm{NE}$ & Int. & Weed/Interfering crops \\
\hline Sonchus asper L. & Shodapay & Th & Mes & $\mathrm{H}$ & $\mathrm{NE}$ & Int. & Interfering crops \\
\hline Tagetes erecta $\mathrm{L}$. & Dambar gulai & Th & $\mathrm{Na}$ & $\mathrm{H}$ & $\mathrm{NE}$ & Int. & Replacing local flora \\
\hline \multicolumn{8}{|l|}{ Adiantaceae } \\
\hline Adiantum capillus-veneris $\mathrm{L}$. & Sumbal & $\mathrm{G}$ & $\mathrm{L}$ & $\mathrm{H}$ & LC & Ind. & - \\
\hline \multicolumn{8}{|l|}{ Apiaceae } \\
\hline Coriandrum sativum $\mathrm{L}$. & Dania & Th & $\mathrm{L}$ & $\mathrm{H}$ & $\mathrm{NE}$ & Int. & - \\
\hline Eryngium bourgatii $\mathrm{L}$. & Azghi & Th & $\mathrm{Mg}$ & $\mathrm{H}$ & $\mathrm{NE}$ & Int. & Interfering local flora \\
\hline \multicolumn{8}{|l|}{ Asclepiadaceae } \\
\hline Calotropis procera (Wild) R. Br. & Spalmay & $\mathrm{Ch}$ & Mes & $\mathrm{H}$ & $\mathrm{NE}$ & Int. & Interfering local flora \\
\hline \multicolumn{8}{|l|}{ Bignoniaceae } \\
\hline $\begin{array}{l}\text { Heterophragma adenophyllum (Wall. ex G. } \\
\text { Don) Seem. ex Benth. \& Hook. f. }\end{array}$ & - & Mesp & Meg & $\mathrm{T}$ & NE & Int. & Interfering local flora \\
\hline \multicolumn{8}{|l|}{ Bombaceae } \\
\hline Bombax ceiba L. & Sumbal & $\mathrm{Np}$ & Mes & $\mathrm{T}$ & $\mathrm{NE}$ & Int. & Interfering local flora \\
\hline \multicolumn{8}{|l|}{ Boraginaceae } \\
\hline Heliotropium europaeum $\mathrm{L}$. & Gulgulay & Th & $\mathrm{Na}$ & $\mathrm{H}$ & $\mathrm{NE}$ & Ind. & - \\
\hline
\end{tabular}




\begin{tabular}{|c|c|c|c|c|c|c|c|}
\hline Division\Family\Species & $\begin{array}{c}\text { Common } \\
\text { Name }\end{array}$ & Life form & Leaf Size & Habit & $\begin{array}{c}\text { Conservation } \\
\text { status }\end{array}$ & $\begin{array}{c}\text { Status } \\
\text { Ind./Int. }\end{array}$ & Impacts \\
\hline \multicolumn{8}{|l|}{ Brassicaceae } \\
\hline Eruca sativa Mill. Gard. & Jamama & Th & Mic & $\mathrm{H}$ & $\mathrm{NE}$ & Int. & Interfering local flora \\
\hline \multicolumn{8}{|l|}{ Cannaceae } \\
\hline Canna indica Linn. & Tasfa botay & $\mathrm{G}$ & Meg & $\mathrm{H}$ & $\mathrm{NE}$ & Ind. & - \\
\hline \multicolumn{8}{|l|}{ Cactaceae } \\
\hline Opuntia littoralis (Engelm) Cockerell & Zaqqqum & $\mathrm{Np}$ & $\mathrm{L}$ & $\mathrm{S}$ & $\mathrm{LC}$ & Int. & Interfering local flora \\
\hline \multicolumn{8}{|l|}{ Cucurbitaceae } \\
\hline Cucurbita pepo L. & Pitta kado & Th & $\mathrm{Mg}$ & $\mathrm{H}$ & $\mathrm{NE}$ & Int. & - \\
\hline Momordica charantia L. & Karela & Th & Mes & $\mathrm{H}$ & $\mathrm{NE}$ & Int. & - \\
\hline \multicolumn{8}{|l|}{ Euphorbiaceae } \\
\hline Euphorbia prostrata L. & Warmagha & Th & $\mathrm{L}$ & $\mathrm{H}$ & $\mathrm{NE}$ & Int. & Interfering local flora \\
\hline \multicolumn{8}{|l|}{ Fabaceae } \\
\hline Cassia fistula L. & Lamdes & $\mathrm{Np}$ & Mes & $\mathrm{T}$ & $\mathrm{NE}$ & Ind. & - \\
\hline Vicia sativa $\mathrm{L}$. & Chilo & Th & $\mathrm{N}$ & $\mathrm{H}$ & $\mathrm{NE}$ & Ind. & - \\
\hline Indigofera heterantha $\mathrm{L}$. & Ghoreeja & $\mathrm{Ch}$ & $\mathrm{N}$ & $\mathrm{H}$ & $\mathrm{NE}$ & Ind. & - \\
\hline Pongamia pinnata (L.) Pierre & Sukh chain & $\mathrm{Np}$ & Mes & $\mathrm{T}$ & $\mathrm{LC}$ & Int. & Interfering local flora \\
\hline Bauhinia variegata (L.) Benth & Kulyar & $\mathrm{Np}$ & Mes & $\mathrm{T}$ & $\mathrm{NE}$ & Ind. & - \\
\hline \multicolumn{8}{|l|}{ Lamiaceae } \\
\hline Mentha arvensis L. & Podina & $\mathrm{G}$ & Mic & $\mathrm{H}$ & $\mathrm{NE}$ & Ind. & - \\
\hline Ocimum basilicum $\mathrm{L}$. & Kashmala & $\mathrm{Ch}$ & Mic & $\mathrm{H}$ & $\mathrm{NE}$ & Ind. & - \\
\hline \multicolumn{8}{|l|}{ Moraceae } \\
\hline Broussonetia papyrifera (L.) Vent & Gulthoothe & Mesp & Mes & $\mathrm{T}$ & $\mathrm{NE}$ & Ind. & - \\
\hline Ficus carica Hausslen. ex. Boiss & Enzar & $\mathrm{Np}$ & Mes & $\mathrm{T}$ & $\mathrm{LC}$ & Ind. & - \\
\hline Ficus religiosa $\mathrm{L}$. & Peeple & $\mathrm{Np}$ & Mes & $\mathrm{T}$ & $\mathrm{NE}$ & Ind. & - \\
\hline Morus alba L. & Spin tooth & Mesp & Mes & $\mathrm{T}$ & $\mathrm{NE}$ & Ind. & - \\
\hline \multicolumn{8}{|l|}{ Myrtaceae } \\
\hline Callistemon lanceolatus L. & Bottle brush & Th & $\mathrm{Mg}$ & $\mathrm{T}$ & $\mathrm{NE}$ & Int. & Interfering local flora \\
\hline Melaleuca bracteata L. & - & $\mathrm{Np}$ & Mic & $\mathrm{T}$ & $\mathrm{NE}$ & Int. & Interfering local flora \\
\hline Psidium guajava $\mathrm{L}$. & Amrood & $\mathrm{Np}$ & Mes & $\mathrm{T}$ & $\mathrm{LC}$ & Int. & - \\
\hline \multicolumn{8}{|l|}{ Meliaceae } \\
\hline Melia azedarach L. & Shandai & Mesp & Mes & $\mathrm{T}$ & $\mathrm{NE}$ & Int. & Interfering local flora \\
\hline
\end{tabular}




\begin{tabular}{|c|c|c|c|c|c|c|c|}
\hline Division\Family\Species & $\begin{array}{c}\text { Common } \\
\text { Name }\end{array}$ & Life form & Leaf Size & Habit & $\begin{array}{c}\text { Conservation } \\
\text { status }\end{array}$ & $\begin{array}{c}\text { Status } \\
\text { Ind./Int. }\end{array}$ & Impacts \\
\hline \multicolumn{8}{|l|}{ Malvaceae } \\
\hline Hibiscus rosa-sinensis L. & Hibiscus & $\mathrm{Np}$ & Mes & $\mathrm{S}$ & $\mathrm{NE}$ & Int. & Interfering local flora \\
\hline \multicolumn{8}{|l|}{ Nyctiginaceae } \\
\hline Bougamvillea glabra Choisy & Bengi boti & $\mathrm{Np}$ & Mic & $\mathrm{T}$ & $\mathrm{NE}$ & Int. & Interfering local flora \\
\hline Mirabilis jalapa L. & Gulabasi & Th & Mes & $\mathrm{H}$ & $\mathrm{NE}$ & Int. & Interfering local flora \\
\hline \multicolumn{8}{|l|}{ Oleaceae } \\
\hline Jasminum sambac Aito & Yasmeen & $\mathrm{Ch}$ & Mic & $S$ & $\mathrm{NE}$ & Int. & Interfering local flora \\
\hline Olea ferruginea Royle & Zaitoon/Khona & $\mathrm{Np}$ & Mic & $\mathrm{T}$ & $\mathrm{NE}$ & Ind. & - \\
\hline \multicolumn{8}{|l|}{ Oxalidaceae } \\
\hline Oxalis corniculata L. & Threwakay & Th & $\mathrm{L}$ & $\mathrm{H}$ & $\mathrm{NE}$ & Int. & Interfering local flora \\
\hline \multicolumn{8}{|l|}{ Papaveraceae } \\
\hline Papaver rhoeas $\mathrm{L}$. & Alak jinai & Th & Mic & $\mathrm{H}$ & $\mathrm{NE}$ & Ind. & - \\
\hline Papaver somniferum $\mathrm{L}$. & Doda & Th & Mic & $\mathrm{H}$ & $\mathrm{NE}$ & Ind. & - \\
\hline \multicolumn{8}{|l|}{ Rosaceae } \\
\hline Eriobotrya japonica (Thunb.). Lindley & Lokat & Mesp & Mac & $\mathrm{T}$ & $\mathrm{NE}$ & Int. & - \\
\hline Rosa indica Lindl. & Gulab & $\mathrm{Ch}$ & Mic & $\mathrm{S}$ & $\mathrm{NE}$ & Int. & Interfering local flora \\
\hline \multicolumn{8}{|l|}{ Rhamnaceae } \\
\hline Ziziphus mauritiana Lam. & Mada Bera & Mesp & $\mathrm{N}$ & $\mathrm{T}$ & $\mathrm{LC}$ & Ind. & - \\
\hline $\begin{array}{l}\text { Ziziphus nummularia (Turm. f.) Weight \& } \\
\text { Arn } \\
\end{array}$ & Karkana & $\mathrm{Np}$ & Mic & $S$ & NE & Ind. & - \\
\hline Ziziphus jujuba Mill. & Bera & $\mathrm{Np}$ & Mic & $\mathrm{T}$ & $\mathrm{NE}$ & Ind. & - \\
\hline \multicolumn{8}{|l|}{ Rutaceae } \\
\hline Citrus limon (L.) Osbeck & Neembo & $\mathrm{Np}$ & Mes & $\mathrm{T}$ & $\mathrm{NE}$ & Ind. & - \\
\hline \multicolumn{8}{|l|}{ Salicaceae } \\
\hline Populus ciliata Wall. ex Royle & Zangali sufaida & Mesp & Mes & $T$ & $\mathrm{NE}$ & Ind. & - \\
\hline Populus nigra L. & Sperdar & Mesp & Mes & $\mathrm{T}$ & $\mathrm{LC}$ & Int. & Interfering local flora \\
\hline Salix acmophylla Boiss & Kharwala & Mesp & Mes & $\mathrm{T}$ & $\mathrm{NE}$ & Ind. & - \\
\hline \multicolumn{8}{|l|}{ Solanaceae } \\
\hline Datura innoxia Mill. & Batora & Th & Mes & $\mathrm{H}$ & $\mathrm{NE}$ & Int. & Weed/Interfering crops \\
\hline Datura metel L. & Ghatta batora & Th & $\mathrm{Mg}$ & $\mathrm{S}$ & $\mathrm{NE}$ & Int. & Weed/Interfering crops \\
\hline Datura stramonium L. & Batora & Th & Mes & $\mathrm{H}$ & $\mathrm{NE}$ & Int. & Weed/Interfering crops \\
\hline
\end{tabular}


Ethnobotany Research and Applications

\begin{tabular}{|c|c|c|c|c|c|c|c|}
\hline Division\Family\Species & $\begin{array}{c}\text { Common } \\
\text { Name }\end{array}$ & Life form & Leaf Size & Habit & $\begin{array}{c}\text { Conservation } \\
\text { status }\end{array}$ & $\begin{array}{c}\text { Status } \\
\text { Ind./Int. }\end{array}$ & Impacts \\
\hline \multicolumn{8}{|l|}{ Sapindaceae } \\
\hline Dodonea viscosa (L.) Jacq. & Ghwarskay & $\mathrm{Np}$ & Mic & $\mathrm{S}$ & $\mathrm{NE}$ & Ind. & - \\
\hline \multicolumn{8}{|l|}{ Simaroubaceae } \\
\hline Alianthus altissima (Mill.) Swingle & $\begin{array}{c}\text { Angrezi } \\
\text { Bakayan }\end{array}$ & Mesp & Mic & $\mathrm{T}$ & EN & Int. & Replacing local flora \\
\hline \multicolumn{8}{|l|}{ Tamaricaceae } \\
\hline Tamarix indica Willd. & Beng & $\mathrm{Np}$ & $\mathrm{Na}$ & $\mathrm{T}$ & $\mathrm{NE}$ & Ind. & - \\
\hline \multicolumn{8}{|l|}{ Verbenaceae } \\
\hline Duranta repens L. & Garranta & $\mathrm{Np}$ & Mac & $\mathrm{T}$ & $\mathrm{NE}$ & Int. & Replacing local flora \\
\hline Lantana camara L. & Nagh phool & $\mathrm{Ch}$ & Mic & $\mathrm{S}$ & $\mathrm{NE}$ & Int. & Replacing local flora \\
\hline Verbena hastata $\mathrm{L}$. & Jeeshay & Th & Mic & $\mathrm{H}$ & $\mathrm{NE}$ & Int. & Replacing local flora \\
\hline \multicolumn{8}{|l|}{ Zygophyllaceae } \\
\hline Peganum harmala L. & Spelanay & Hem & Mic & $\mathrm{H}$ & $\mathrm{NE}$ & Ind. & - \\
\hline
\end{tabular}

Key: $\mathrm{T}=$ Tree, $\mathrm{S}=$ Shrub, $\mathrm{H}=$ Herb, $\mathrm{Cl}=$ Climber, $\mathrm{Th}=$ Therophyte, $\mathrm{Mp}=$ Microphanerophyte, Mesp= Mesophanerophyte, Np= Nanophanerophyte, He= Hemicryptophyte, Ph= Phanerophyte, $\mathrm{Ch}=$ Chamaephyte, $\mathrm{Na}=$ Nanophyll, Mes= Mesophyll, Mac= Macrophyll, Mic= Microphyll, Lp= Leptophyll, Aph= Aphyllous; Ind.= Indigenous, Int.= Introduced 


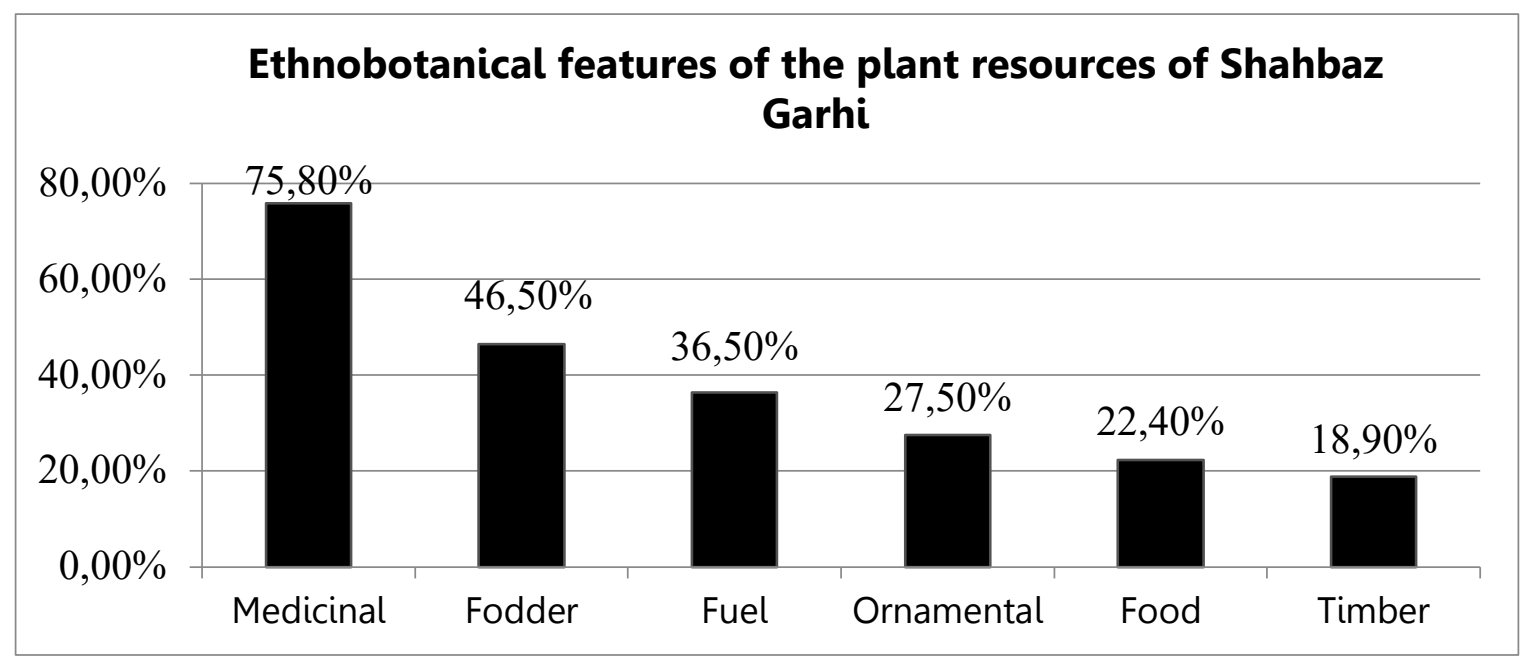

Figure 4. Ethnobotanical assessment of plant growing in the research area.

During the present research 85 species were collected and the findings were compared with some previous researchers who carried out, similar studies on the surrounding areas in similar climatic areas like Akhtar \& Begum (2009) carried out the survey of plants of Jalala district Mardan. They identified 55 plants belonging to 38 families. Studies on floristic composition of district Mardan were conducted and 91 plant species belonging to 76 genera and 38 families were identified by (Khan et al. 2013) and noted that Asteraceae was the dominant family which shows similarity with this research. In a study from Torghar 25 species of Asteraceae and 21 species of Poaceae were reported by (Mehmood et al. 2015) which resembles our results. (Malik et al. 2004) These families were also well represented in Kotli Hill during monsoon by (Malik et al. 2004). Asteraceae and Poaceae families were also observed dominant by (Durrani et al. 2005) in their research areas.

Similar work was carried out on the floristic composition and ecological characteristics of Karkhasa, Balochistan, Pakistan which consisted of 154 plant species belonging to 39 families by (Shaista et al. 2020). The floristic composition and biological spectrum of Rangeland, District Tank Pakistan was studied by (Lal et al. 2013). There were 205 species belonging to 56 families. Acacia modesta, Achyranthus aspera, Calotropis procera, Opuntia littoralis, Broussonetia papyrifera, Ficus carica, Morus alba, Eucalyptus lanceolatus, Melia azedarach, Ficus religiosa, Prunus persica, Ailanthus altissima and Tamarix indica were commonly found. Our result is also similar with that of (Khan et al. 2012). The dominance of Therophytes occurs due to un-favorable environmental conditions (Shimwell 1971). Chamaephytes and Therophytes were considered major life form in unfavorable environment in desert region by (Khan et al. 2012).

Study was made on Sathan Galli, District Mansehra of Khyber Pakhtunkhwa (KP), Pakistan and 55 families were reported by (Khan et al. 2017). Leaf size spectra were dominated by Microphyll contributing 68 species which resembles our results. Therophytes were found as leading life also contributing to our research. Microphylls are usually characteristics of steppes while Nanophylls and Leptophylls are characteristics of hot deserts (Khan et al. 2013). The dominance of Therophytes was also showed from Tehsil Katlang, District Mardan by (Khan et al. 2014) which prove that the area is under heavy biotic stress. Therophytes and Microphylls are dominant life form and leaf size of the Cholistan Desert, Pakistan (Wariss et al. 2013). Therophytes were also reported as most dominant class in Hayatabad, Peshawar by (Shah and Hussain 2009).

The medicinal plants of Tehsil Kabal District Swat, KP, Pakistan was studied, and 45 plants were identified, among them, 30 were herbs, followed by 13 shrubs and 2 were trees which shows the same results to our research (Khan et al. 2015). The ethnobotanical studies on plant resources of Sheikh Maltoon, District Mardan were conducted by (Khan \& Musharaf 2014). They reported 73 medicinal plants species. The medicinal floral knowledge of Sarban Hills, Abbottabad, Khyber Pakhtunkhwa, Pakistan reported 74 plant species belonging to 70 genera and 42 families (ljaz et al. 2016). Information on the uses of various plants of District Tank found that 41 plant species were commonly used by local people for curing various diseases (Badshah and Hussain 2011). 


\section{Conclusion and Recommendations}

It was concluded during the present research that the area has a rich phytodiversity and 85 species were recorded during the present research. People of the area use these plants for different purposes. After the critical analysis of the phytodiversity and ethnobotanical evaluation, it was noted that the local inhabitants of the area relay upon plants for fulfilling their daily needs. The inhabitants obtained their dairy products from domestic animals including buffalos, cows and goats. They feed their cattle by using aerial parts of the plants including leaves and buds of plants such as Aerva javanica, Adiantum capillus-veneris and Ailanthus altissima. Many species such as Conyza canadensis, Calotropis procera and Canna indica are used for curing various diseases. Since there is no alternative source available of fuel in the area therefore, the locals depend upon the fuel wood for heating and cooking purposes which includes Bombax ceiba, Bauhinia vareigata and Broussonetia papyrifera. Many species such as Cassia fistula and Morus alba are used for construction purposes thus the locals also extract timber wood from the tree species growing in the area. Food is the basic need of human being and in the research area many species are used as food including Allium sativum, Coriandrum sativum, Citrus limon and Ficus carica. The plants such as Halianthus annus, Jasminum sambac, Jasminum humile, Morus alba, Nerium oleanderand Rosa indica are also used for beautification purposes and some revenue is generated by the local inhabitants by selling some of the ornamental plants. The present research showed that plants play an important role in supporting the livelihood of the local people. Plants not only provide fodder, medicine, fuel wood, timber and food but are also used as ornamentals and thus the plants support the food security directly and indirectly support the livelihood by providing economic security.

It is recommended that there is a potential for commercialization of many species like Heterophragma adenophyllum, Pinus roxburghii and Melia azedarach for the purpose of furniture Industry. Similarly, the fuel wood species can be grown and adopted as farm forestry species for generating revenue to support the livelihood of the people. It is also recommended that awareness must be brought among the residents of the area for sustainable utilization of the species. Overexploitation of the plants growing in the area may cause vanishing of many indigenous plant species.

\section{Declarations}

List of abbreviations: T-Tree, S-Shrub, H- Herb, Cl-Climber, Th-Therophytes, Mp- Microphanerophyte, MespMesophanerophyte, Np- Nanophanerophyte, He- Hemicryptophyte, Ph-Phanerophyte, Ch- Chamaephyte, NaNanophyll, Mes- Mesophyll, Mac-Macrophyll, Mic- Microphyll, Lp-Leptophyll, Aph- Aphyllous, Fd- Fodder, MdMedicinal, Fu- Fuel, Tm- Timber, Fod- Food, Orn- Ornamental, += Yes, - = No.

Ethics approval and consent to participate: No ethical approval is involved in the current research work. Availability of data and materials:

The dried plant specimens were mounted on standard herbarium sheets and were deposited in the Herbarium of Department of Botany, University of Peshawar (PUP).

Competing interests: The authors have no competing interests.

Funding: No funding was received for the present research study.

Author's contribution: Sadia Parveen made visits to the research area for collection of plants and other relevant data. Lal Badshah helped in the selection of research topic and identification of ecological characteristics. Asad Ullah helped in identification of plants, in formatting and sorting of paper. Syed Ghias Ali and Usman Ali helped in processing, mounting of specimens and typographic correction in the paper. Syed Mukaram Shah helped in ecological data collection and proof reading. Akhtar Zaman had made contribution in identification. Sara Hamid Siddiqui accompanied the principal author during field visits and helped in collection of specimens.

\section{Literature Cited}

Ahmad F. 2007. Geo-informatics application to investigate agricultural potential in Cholistan desert. Journal of Food Agriculture \& Environment 5:310-14.

Ahmad L, Riaz M, Jan HA, Semotiuk AJ, Ahmad I, Khan I, Ali F, Rashid W, Bussmann RW. 2021. An ethnobotanical survey of wild food plants used by the local communities of Kumrat Valley in District Dir Upper, Pakistan. Ethnobotany Research and Applications 22(20):1-13.

Ahmed W, Qureshi R, Arshad M. 2019. Floristic, frequency and vegetation biological spectra of Murree-Kotli SattianKahuta National Park, Pakistan. Pakistan Journal of Botany 51(2):637-648. 
Ajaib M, Ishtiaq M, Bhatti KH, Hussain I, Maqbool M, Hussain T, Waheed M, Ghani A, Azeem M, Khan SMR, Thind S, Bashir R. 2021. Inventorization of traditional ethnobotanical uses of wild plants of Dawarian and Ratti Gali areas of District Neelum, Azad Jammu and Kashmir, Pakistan. Ethnobotany Research and Applications 16(7):1-34.

Akhtar N, Begum S. 2009. Ethnopharmacological important plants of Jalala, district Mardan, Pakistan. Pakistan Journal of Plant Sciences 15(2):95-100.

Ali H, Qaiser M. 2009. The Ethnobotany of Chitral Valley, Pakistan with particular reference to medicinal plants. Pakistan Journal of Botany 41(4):2009-2041.

Ali M, Aldosari A, Tng DYP, Ullah M, Hussain W, Ahmad M, Hussain J, Khan A, Hassan HH, Bussman SRW, Shao JW. 2019. Traditional uses of plants by Indigenous communities for Veterinary practices at Kurram District, Pakistan. Ethnobotany Research and Applications 18(24):1-19.

Ali SI, Nasir YJ. 1989-1991. Flora of Pakistan. Department of Botany, Karachi University Karachi. 191-193.

Ali SI, Qaiser M. 1993-2021. Flora of Pakistan. Department of Botany, Karachi University Karachi. 194-221.

Ali SI. 2008. The significance of flora with special reference to Pakistan. Pakistan Journal of Botany 40(30): 967-971.

Badshah L, Hussain F, Sher Z. 2013. Floristic inventory, ecological characteristics and biological spectrum of rangeland, District Tank, Pakistan. Pakistan Journal of Botany 45(4):1159-1168.

Badshah L, Hussain F. 2011. People preferences and use of local medicinal flora in District Tank, Pakistan. Journal of Medicinal Plants Research 5(1):22-29.

Durrani JM, Hussain F. 2005. Ecological characteristics of plants of Harboi rangeland, Kalat, Pakistan. Journal of Tropical and Subtropical Botany 13(2):130-138.

Harshberger JW. 1896. The purposes of ethnobotany. Botanical Gazette 21: 146-154.

Hussain F. 1989. Field and Laboratory Manual of Plant Ecology. UGC. Islamabad.

ljaz F, Iqbal Z, Rahman IU, Khan SM, Shah GM, Afzal A. 2016. Investigation of traditional medicinal floral knowledge of Sarban Hills, Abbottabad, KP, Pakistan. Journal of Ethnopharmacology 179:208-233.

Ilyas M, Qureshi R, Arshad M, Mirza SN. 2013. A preliminary chick list of the vascular Flora of Kabal Valley, Swat, Pakistan. Pakistan Journal of Botany 45:605-615.

IUCN. Species Survival Commission [Online]. 2019. Version 14. Available from http://intranet.iucn.org/webfiles/doc/SSC/RedList/RedListGuidlines.pdf (accessed 30/9/2021).

Jan M, Mir TA, Ganie AH, Khare RK. 2021. Ethnomedicinal use of some plant species by Gujjar and Bakerwal community in Gulmarg Mountainous Region of Kashmir Himalaya. Ethnobotany Research and Applications 21(38):1-23.

Khan M, Hussain F, Faridullah, Musharaf S. 2014. Classification of plant species in Tehsil Katlang, District Mardan, Khyber Pakhtunkhawa, Pakistan. Journal of Medicinal Plant Research 4(3):18-29.

Khan M, Hussain F, Musharaf S. 2012. Degree of Homogeneity of Plant Life in Tehsil Takht-e-Nasrati, Pakistan. Global Journal of Science Frontier Research 12(4):65-72.

Khan M, Hussain F, Musharaf S. 2012. Degree of Homogeneity of Plant Life in Tehsil Takht-e-Nasrati, Pakistan. Global Journal of Science Frontier Research 12(4):65-72.

Khan M, Hussain F, Musharaf S. 2013. Floristic Composition and Biological Characteristics of the Vegetation of Sheikh Maltoon Town District Mardan, Pakistan. Annual Review \& Research in Biology 3(1):31-41.

Khan M, Hussain F, Musharaf S. 2013. Floristic composition and biological characteristics of the vegetation of Sheikh Maltoon Town District Mardan, Pakistan. Annual Research \& Review in Biology 3(1):31-41.

Khan M, Hussain F, Musharaf S. 2014. Floristic Composition and Ecological Characteristics of Shahbaz Garhi, District Mardan, Pakistan. Global Journal of Science Frontier Research 1:7-1.

Khan M, Ishtiaq KR, Iqbal Z, Alam J, Shah AH, Farooq M. 2017. Biological spectra of vegetation of Sathan Gali, Mansehra, KPK, Pakistan. World Scientific News 87:136-149.

Khan S, Sohail NU, Rahman Fl, Iqbal Z, Ali Z 2015. Ethnobotanical study of some medicinal plants of Tehsil Kabal, District Swat, KP, Pakistan. Journal of Medicinal and Aromatic Plants 4(189):2167-0412.

Kharkwal G, Mehrotra P, Pangley YPS. 2005. Phytodiversity and growth form in relation to altitudinal gradient in the Central Himalayan (Kumaun) region of India. Current Science (89):873-878.

Malik NZ, Malik ZH. 2004. Present status of subtropical chir pine vegetation of Kotli Hills, Azad Jammu and Kashmir. Journal of Research in Science 15(1):85-90.

Martin GJ. 2004. Ethnobotany. 8-12 Camden High Street London, NW 10 JH, UK. pp 67-116. 
Mehmood A, Khan SM, Shah AH, Ahmad H. 2015. First floristic exploration of the District Torghar, Khyber Pakhtunkhwa, Pakistan. Pakistan Journal of Botany 47 (SI):57-70.

Mukerjee PK, Wahil A. 2006. Integrated approaches towards drug development from Ayurveda and other Indian system of medicine. Journal of Ethnopharmacology 103:25-35.

Nasir E, Ali SI. 1970-1989. Flora of Pakistan. Department of Botany, University of Karachi, Karachi. 1-190.

Qureshi R, Waheed A, Arshad M. 2009. Weed communities of wheat crop in district Toba Tek Singh, Pakistan. Pakistan Journal of Botany 41:239-245.

Raunkiaer, C 1934. Life form of plants and statistical plant geography. Clarendon Press Oxford.

Shah M, Hussain F. 2009. Phytosociological study of the vegetation of Hayat Abad Peshawar, Pakistan. Pakistan Journal of Plant Sciences 15(2).

Shaista A, Hussain F, Durrani MJ, Masood A, Mushtaq A, Rizwan S, Behlil F. 2020. Floristic Composition, Ecological characteristics and Ethnobotanical profile of protected and open grazing land of Karkhasa, Balochistan. Pakistan Journal of Animal and Plant Sciences 30(2):420-430.

Sher Z, Hussain F, Badshah L, Wahab M. 2011. Floristic composition, communities and ecological characteristics of weeds of wheat fields of Lahore, District Swabi, Pakistan. Pakistan Journal of Botany 43:2817-2820.

Shimwell DW. 1971. The Description and Classification of Vegetation Sedgwick and Jackson, London: 322.

Shinwari, ZK, Gilani SS. 2003. Sustainable harvest of medicinal plants at Bulashbar Nullah, Astore (northern Pakistan). Journal of Ethnopharmacology 84:289-98.

Stewart RR. 1972. An annotated catalogue of the vascular plants of West Pakistan and Kashmir, Fakhri Printing Press, Karachi

Stijhoorn, E. (1996-97). New timber forest products and Ethnobotany, concepts for management and research Department of Environment and Plant Biology, Ohio University, author, Ohio/ LATIE, Turrialba, 32.

Ullah A. 2014. Biodiversity and Ethnobotanical study of Vascular Plants in Mankial Valley Hindukush Range Pakistan. Ph.D. thesis. Department of Botany, University of Peshawar.

Waris H, Mukhtar M, Anjum S, Bhatti GR, Pirzada SA, Alam K. 2013. Floristic Composition of the Plants of the Cholistan Desert, Pakistan. American Journal of Plant Sciences 4(12):58-65. 